\title{
Genomic insight into the origins and evolution of symbiosis genes in Phaseolus vulgaris microsymbionts
}

Wenjun Tong ${ }^{1 \dagger}$, Xiangchen $\mathrm{Li}^{1,2 \dagger}$, Entao Wang ${ }^{3}$, Ying Cao ${ }^{1}$, Weimin Chen ${ }^{1 *}$, Shiheng Tao ${ }^{2^{*}}$ and Gehong Wei ${ }^{1^{*}}$ (D)

\begin{abstract}
Background: Phaseolus vulgaris (common bean) microsymbionts belonging to the bacterial genera Rhizobium, Bradyrhizobium, and Ensifer (Sinorhizobium) have been isolated across the globe. Individual symbiosis genes (e.g., nodC) of these rhizobia can be different within each genus and among distinct genera. Little information is available about the symbiotic structure of indigenous Rhizobium strains nodulating introduced bean plants or the emergence of a symbiotic ability to associate with bean plants in Bradyrhizobium and Ensifer strains. Here, we sequenced the genomes of 29 representative bean microsymbionts (21 Rhizobium, four Ensifer, and four Bradyrhizobium) and compared them with closely related reference strains to estimate the origins of symbiosis genes among these Chinese bean microsymbionts.
\end{abstract}

Results: Comparative genomics demonstrated horizontal gene transfer exclusively at the plasmid level, leading to expanded diversity of bean-nodulating Rhizobium strains. Analysis of vertically transferred genes uncovered 191 (out of the 2654) single-copy core genes with phylogenies strictly consistent with the taxonomic status of bacterial species, but none were found on symbiosis plasmids. A common symbiotic region was wholly conserved within the Rhizobium genus yet different from those of the other two genera. A single strain of Ensifer and two Bradyrhizobium strains shared similar gene content with soybean microsymbionts in both chromosomes and symbiotic regions.

Conclusions: The 19 native bean Rhizobium microsymbionts were assigned to four defined species and six putative novel species. The symbiosis genes of R. phaseoli, R. sophoriradicis, and R. esperanzae strains that originated from Mexican bean-nodulating strains were possibly introduced alongside bean seeds. $R$. anhuiense strains displayed distinct host ranges, indicating transition into bean microsymbionts. Among the six putative novel species exclusive to China, horizontal transfer of symbiosis genes suggested symbiosis with other indigenous legumes and loss of originally symbiotic regions or non-symbionts before the introduction of common bean into China. Genome data for Ensifer and Bradyrhizobium strains indicated symbiotic compatibility between microsymbionts of common bean and other hosts such as soybean.

Keywords: Phaseolus vulgaris, Horizontal gene transfer, Vertical gene transfer, Comparative genomics, Symbiosis genes

\footnotetext{
* Correspondence: chenwm029@nwafu.edu.cn; shihengt@nwafu.edu.cn; weigehong@nwafu.edu.cn

${ }^{\dagger}$ Wenjun Tong and Xiangchen Li contributed equally to this work.

${ }^{1}$ State Key Laboratory of Crop Stress Biology for Arid Areas, Shaanxi Key Laboratory of Agricultural and Environmental Microbiology, College of Life Science, Northwest A\&F University, Yangling, Shaanxi 712100, People's Republic of China

${ }^{2}$ Bioinformatics Center, Northwest A\&F University, Yangling, Shaanxi 712100

People's Republic of China

Full list of author information is available at the end of the article
}

(c) The Author(s). 2020 Open Access This article is distributed under the terms of the Creative Commons Attribution 4.0 International License (http://creativecommons.org/licenses/by/4.0/), which permits unrestricted use, distribution, and reproduction in any medium, provided you give appropriate credit to the original author(s) and the source, provide a link to the Creative Commons license, and indicate if changes were made. The Creative Commons Public Domain Dedication waiver (http://creativecommons.org/publicdomain/zero/1.0/) applies to the data made available in this article, unless otherwise stated. 


\section{Background}

Most legumes can establish mutualistic symbiosis with certain Alphaproteobacteria or Betaproteobacteria known as rhizobia [1]. Such symbiotic relationships have coevolved over millions of years and are fundamental to sustainable agriculture because they contribute approximately half of global terrestrial nitrogen nutrients [2]. Phaseolus vulgaris L. (common bean) is an important leguminous food crop cultivated worldwide in a broad range of cropping systems and environments. This species was domesticated from a wild-growing vine around 7000 years ago, in two primary centers of origin located in Mexico/Central America and the southern Andes (Ecuador, Peru, Chile, and Argentina) [3, 4]. Like many other legumes, common bean plants form efficient nitrogen-fixing nodules with diverse rhizobia [5-7]. To date, 18 Rhizobium species have been isolated from common bean root nodules. In addition, the symbiovar (sv.) mediterranense in Ensifer (Sinorhizobium) meliloti [8], E. fredii [9], and E. americanum [10] can nodulate common bean plants in alkaline-saline soils. Some of these rhizobia have been detected in both the centers of origin and other areas because they can be introduced with common bean seeds [11].

Common bean is believed to have been introduced into China directly from Latin America around 400 years ago [12], and China is now one of the world's major producers of common bean. In previous studies, 371 rhizobial strains were isolated from root nodules of common bean plants grown in fields at 21 sample sites in four provinces in China $[13,14]$. Approximately $89 \%$ of these isolates were Rhizobium, including six defined species and eight novel genospecies, while the remaining strains were Bradyrhizobium (2.4\%) and Ensifer (8.4\%) [13]. To date, several species such as $R$. anhuiense [15], E. fredii [14], and some genospecies from Bradyrhizobium [13] and Ensifer [14] have only been found in China. Some of these common bean-nodulating rhizobia were also found in association with other hosts, such as Vicia faba (fava bean) and Trifolium spp. (clover) in China [15]. How such an extraordinary variety of genotypes from three distinct genera (Rhizobium, Bradyrhizobium, and Ensifer) evolved into microsymbionts of common bean after its introduction into China is very interesting.

Concerning the location of symbiosis genes (on plasmids or chromosomes), Rhizobium species [16], Mesorhizobium huakuii, M. amorphae [17, 18], and Ensifer species [19] differ from Bradyrhizobium species [20, 21], M. loti [22], and M. ciceri [23]. Growing evidence indicates that horizontal gene transfer (HGT) of symbiosis plasmids/islands allowed diverse bacteria to engage in symbiosis with different leguminous host plants during the evolution of rhizobia [24, 25]. Hosts also play a role in HGT; for example, the roots of Sesbania rostrata secrete flavonoids that induce nodulation in the rhizobium-legume mutualistic symbiosis while enhancing the transfer of Azorhizobium caulinodans symbiosis islands [26]. However, it is unclear whether common bean enhances HGT in rhizobia during its adaptation to the introduced environment. Furthermore, common bean and Glycine max L. (soybean), both members of the Phaseoleae family, diverged 19 million years ago [27]. Soybean is a major leguminous crop originating in East Asia and it has been planted in China for over 5000 years. Different gene pools of soybean and its microsymbionts (mainly Ensifer and Bradyrhizobium strains) have been reported in various ecoregions of China [28, 29]. Therefore, the relationships of symbiosis genes in Ensifer and Bradyrhizobium strains that nodulate common bean and soybean in China are of interest.

Nodule-forming leguminous plants have been divided into three categories based on symbiotic specificities [30, 31]: (1) those stringently selected on both chromosomal and symbiosis gene backgrounds, such as Medicago sativa L. (alfalfa); (2) those stringently selected on symbiosis gene background only, such as common bean, Robinia pseudoacacia L. (black locust) [32], and Aspalathus carnosa [33]; (3) those nodulating with diverse rhizobia harboring different symbiosis genes, such as soybean [29] and Sophora flavescens [31]. Common bean belongs to the secondary category even though three genotypes of rhizobial symbiosis genes (sv. phaseoli, sv. tropici, and sv. mimosa) have been identified [15]. Approximately 20 genospecies in sv. phaseoli (including $R$. etli CFN42 $2^{\mathrm{T}}$ and $R$. esperanzae CNPSo668 ${ }^{\mathrm{T}}$ ) with different chromosomal backgrounds share nodC gene similarities of $97.3-100 \%$ [15]. In contrast, strains in sv. tropici and sv. mimosa harbor symbiotic genes different from those of sv. phaseoli, and these strains have a wide host range $[15,34,35]$. In these cases, symbiosis genes, typically those involved in nodulation (nod, nol, and noe) [36] and nitrogen fixation (nif, fix, and $f d x$ ) [37] in rhizobia, might have been transferred vertically in some species, but horizontally in others [38]. However, a plant introduced into a new environment could acquire indigenous rhizobia originally associated with a native legume species [39].

To clarify how different rhizobial species were recruited as symbionts of common bean in China, comparison of individual symbiosis genes, such as nodC, could provide insight into the phylogenetic relationships [40]. So far, more than 500 genes have been identified to be involved in rhizobium-legume symbiosis (e.g., nod, nif, nol, fix, exo, and lps) [41, 42]. Analysis of individual gene still lacks information on the genetic structure and interactions of symbiosis genes. Fortunately, genomics has revolutionized the way for estimation of the phylogenetic relationships among microbes, including the 
evolution of rhizobia [41]. In particular, comparison of whole genomes could contribute to our understanding about the relationships between rhizobia in the countries of origin and introduced regions.

Herein, we chose 25 representatives of common bean -nodulating rhizobia from China (19 Rhizobium, two Ensifer, and four Bradyrhizobium), and four from Mexico (two Rhizobium and two Ensifer), for comparative genomics analysis with reference strains. The genome analyses could shed light on different genetic associations while fully explaining the genetic structure of rhizobial strains. The goals of this study were (i) to estimate the origins of symbiosis genes among the common bean-nodulating rhizobial strains belonging to Rhizobium, Ensifer, and Bradyrhizobium, and (ii) to gain genomic insight into different symbiovars among the rhizobia investigated.

\section{Results}

\section{Genomic features of core and accessory Rhizobium genomes}

To analyze genomic features among strains in the Rhizobium genus occupying diverse niches, we used the 50 available Rhizobium genomes (Additional file 1: Table S1) to probe the evolution of the gene repertoire through pan-genome analysis. The pan-genome consisted of three parts, the core genome (shared by all strains), the dispensable genome (shared by some but not all strains), and the unique genome (unique to individual strains). The 50 Rhizobium strains were classified into 19 clusters or species (labeled R1 through R19) at the $95 \%$ average nucleotide identity (ANI) threshold for species delineation; this is consistent with the grouping results of digital DNA:DNA hybridization (dDDH) estimation and multilocus sequence analysis of housekeeping genes [15].

To better understand the pan-genome of Rhizobium strains, we clustered 315,181 coding sequences (CDSs) obtained from the 50 available genomes. This yielded a pan-genome containing 30,767 homologous gene families in the genus Rhizobium, with 2777 homologous genes in the core genome and 14,243 genes in the dispensable genome. The core genome represented 39.93 to $47.59 \%$ of the repertoire of protein-coding genes in each strain. Moreover, 13,747 genes belonging to the unique genome represented only one strain of Rhizobium (Fig. 1a). The number of strain-unique genes varied from five (R1-N771, with 6800 CDSs) to 1139 (R19STM6155, with 6561 CDSs). It is noteworthy that all five unique genes in R1-N771 (and all six unique genes in R5-N561) encode hypothetical proteins. Hypothetical protein-coding genes accounted for more than $61 \%$ of unique genes in each strain.

Furthermore, we used hierarchical clustering to construct bifurcating trees and identified strains sharing similar gene content based on the presence and absence of 30,767 genes in the pan-genome across the 50 Rhizobium genomes. The hierarchical cluster derived from these data clearly distinguished strains of the same species from those of different species (Fig. 1b). The
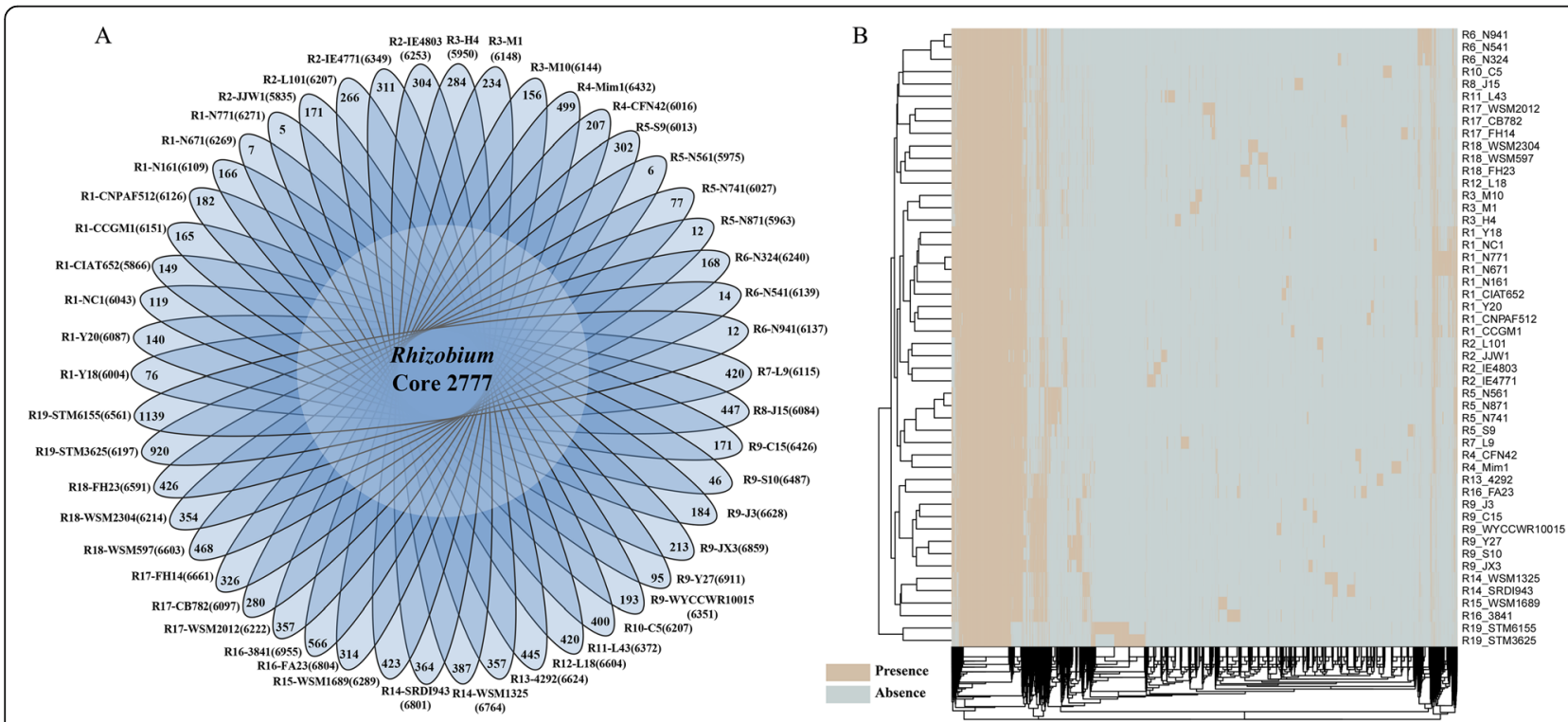

Fig. 1 The pan-genome of 50 Rhizobium strains used in this study. a Flower plot showing the number of strain-specific genes (in petals) and core genes common to all Rhizobium strains (in the center). The name of each strain is preceded by the cluster number indicated in Additional file 1: Table S2. b Hierarchical clustering of Rhizobium genomes based on a heatmap of 30,767 genes in the pan-genome. The presence and absence of the 30,767 genes are indicated by bisque and azure, respectively 
clustering results were well supported by the interspecies assignments based on the neighbor-joining species tree of 2110 concatenated single-copy core genes shared by 50 Rhizobium genomes (Additional file 2: Figure S1).

\section{Species and host trees}

To comprehensively understand the evolution of common bean Rhizobium microsymbionts, we chose 29 representative strains (Additional file 1: Table S1) in 10 genospecies (clusters) which comprised more than two strains each. The representative strains were used for the analysis of vertically transferred genes that could reflect phylogenetic relationships among these strains, and horizontally transferred genes that may be related to host specificity.

A total of 2654 single-copy core genes were extracted from the 29 representative strains and phylogenetic trees were constructed to identify genes supporting the known phylogeny of rhizobia. The Shimodaira-Hasegawa test for the comparison between the phylogenetic tree for each of the 2654 core genes and the species tree uncovered 191 genes with consistent phylogenies (Additional file 1: Table S2). Of these 191 core genes, none were found on symbiosis plasmids, and only five were detected on accessory plasmids, as indicated in the complete genome of $R$. etli CFN42 $2^{\mathrm{T}}$. Specifically, one gene encodes a hypothetical protein on plasmid $\mathrm{p} 42 \mathrm{~b}$, while the other four genes encode a probable transcriptional regulator protein in the IclR family, an oligopeptide $A B C$ transporter substrate-binding protein, an XRE family transcriptional regulator protein, and an oligopeptide $\mathrm{ABC}$ transporter substrate-binding protein, respectively, on plasmid p42e in $R$. etli CFN42 ${ }^{\mathrm{T}}$. Most (186 out of the 191) species-related genes were located on chromosomes. With universal distribution and strictly vertical transfer among the 29 rhizobial genomes, 16 highlyconserved genes encode hypothetical proteins. These 16 genes may perform essential biological functions in the survival of rhizobial strains. In general, 65 genes were related to metabolism (e.g., plsCX, fabAD, metCK, folC, $m g s A$, aglK, purF, serB, $\arg C H$, and $d p p B), 24$ genes were linked to translation and biogenesis (e.g., $\operatorname{mur} B C$, exoN, hisS, rpsK, tlyA, tsf, and frr), 18 genes were associated with transcription (e.g., $\operatorname{csp} B G$ and $n r d R$ ), and eight genes were involved in defense mechanisms and signal transduction mechanisms (e.g., lolD, $m s b A$, pleD, and $d k s A$; Additional file 1: Table S2).

Before identifying the core genes related to symbiotic specificity, we first carried out cross-nodulation tests with four legume species (Trifolium pratense, Mimosa pudica, Phaseolus vulgaris, and Leucaena leucocephala) to verify the symbiotic specificity. The rhizobiumlegume symbiosis was highly specific, such that each rhizobial genus/species/strain could nodulate only a specific group of legume, and vice versa [43]. We then chose seven representative Rhizobium strains from clusters (species) containing the corresponding symbiovars. The results confirmed that all representative strains could nodulate their original host only (Additional file 2: Figure S2). Although R2-L101 possessed two types of nod gene clusters (partial Phaseolus-type and complete Mimosa-type; Additional file 2: Figure S3), this strain could not engage in symbiosis with $M$. pudica or L. leucocephala. Several core genes specifically related to the host of origin were found on symbiosis plasmids. Since symbiosis plasmids/islands can be transferred from an inoculant strain to a non-symbiotic strain, and since symbiotic regions are usually clustered in particular regions, we investigated the nod, nif, and fix gene clusters further. Twelve symbiosis genes (nodABC, fix $A B C$, and nifHDKENB) were found to be related to the host of origin (Additional file 2: Figure S3). These genes represent diverse genomic organization and may act as the major determinants of symbiotic specificity.

\section{Recent HGT among Rhizobium strains}

To investigate HGT events in the Rhizobium genus, we obtained the complete sequences of symbiosis plasmids from $R$. acidisoli FH23 for HGT analysis. The genome size of strain FH23 was 7,497,685 bp (135,772 bp larger than its draft genome), which comprised a chromosome $(4.57 \mathrm{Mb})$ with a $\mathrm{G}+\mathrm{C}$ content of $61.5 \%$ and four plasmids $(0.67-0.85 \mathrm{Mb})$ with a $\mathrm{G}+\mathrm{C}$ content of $58.4-$ $61.1 \%$. Most of the nod, nif, and fix symbiosis genes were clustered in a $0.1 \mathrm{Mb}$ region on symbiosis plasmid pRapFH23a (Additional file 2: Figure S4).

To explore the effects of HGT among the 35 Rhizobium strains nodulating $P$. vulgaris (Additional file 1: Table S1), we employed a pairwise sequence conservation strategy known as RecentHGT [44]. A total of 447 species pairs among the 35 Rhizobium strains were found to share a significant number ( $>50)$ of HGT genes $($ mean $=110$, standard deviation $=39$; Additional file 1 : Table S3). The number of HGT genes shared between some species was considerably large; for example, there were 20 species pairs among R1, R2, R5, R9, R11, R12, and R13, sharing over 200 HGT genes. By contrast, only three species (R14, R15, and R19) shared few recent gene communication events. Moreover, the number of predicted HGT genes had no significant correlation (Spearman's $\rho=-0.057, p=0.58$ ) with the ANI values between the 447 species pairs. The results indicate that phylogenetic distance was not a significant barrier for recent HGT events among Rhizobium species.

Based on the reference genome R4-CFN42, we found that the predicted number of HGT genes and the number of highly conserved homologs on plasmids were 
nearly identical among most of the HGT events between two species (Fig. 2a). In addition, most of the HGT genes were found on symbiosis plasmids, and on accessory plasmid p42a in strains R4 and R17 (R4-CFN4 and R17-FH14; Fig. 2b). Notably, some HGT genes were symbiosis genes (nod/nif/fix) and mobile elements, encoding plasmid proteins with key functions such as (1) replication (e.g., the $\operatorname{rep} A B C$ operon) and (2) conjugation (e.g., type IV secretion system and conjugative transfer relaxase). Moreover, we found that all inferred HGT events occurred within the Rhizobium species isolated from $P$. vulgaris root nodules only, and ANI values

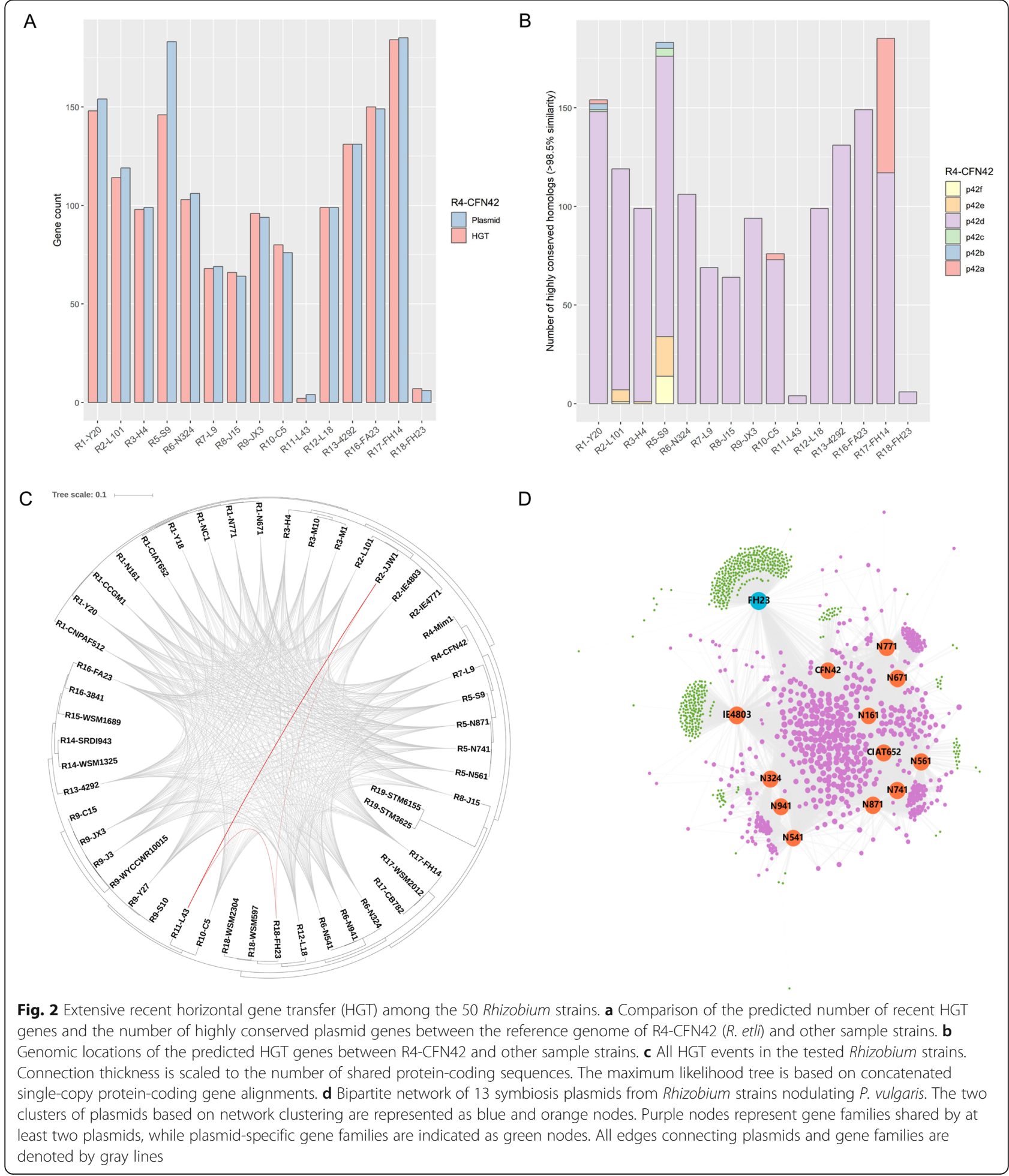


of some species pairs from different host plants were considerably smaller (Additional file 1: Table S3). Hostspecific HGT events were consistent with host selection of symbiosis genes.

It is worth noting that two different HGT groups were identified based on the occurrence of HGT events among the 35 Rhizobium strains nodulating $P$. vulgaris (Fig. 2c), and there was a small group of only three strains (R2-JJW1, R11-L43, and R18-FH23). To explore the phylogenetic relationship of symbiosis plasmids in the two HGT groups, we constructed a bipartite 'gene families-plasmids' network of 13 symbiosis plasmids from the Rhizobium strains nodulating $P$. vulgaris, in which one partition represented plasmids and the other represented homologous gene families (Fig. 2d). We then performed a hierarchical clustering analysis and identified plasmid clusters at a 95\% distance threshold. The network revealed that the symbiosis plasmid in R18-FH23 was distant from other symbiosis plasmids, sharing fewer homologous genes and containing more unique genes. Further, we analyzed functional enrichment of these unique genes using clusters of orthologous groups (COG) annotations (Additional file 2: Figure S5). Apart from the 'general function prediction only' category, unique genes were significantly enriched in 'inorganic ion transport and metabolism' and 'amino acid transport and metabolism' categories (Fisher's exact test, $p<0.01$ ). In summary, symbiosis plasmids in R2-JJW1,
R11-L43, and R18-FH23 were substantially different from other symbiosis plasmids with regard to $P$. vulgaris-Rhizobium symbiosis.

\section{Relationships between microsymbionts of common bean and other hosts}

In the genome analysis of four Ensifer and four Bradyrhizobium microsymbionts of common bean, we obtained six and 13 related reference genomes from Genbank, respectively (Additional file 1: Table S2). At the 95\% ANI threshold for species delineation, the 10 Ensifer strains and 17 Bradyrhizobium strains were divided into five and eight clusters, respectively, with an average aligned percentage of $85.88 \%$. The result was consistent with their evolutionary relationships based on MAUVE alignments (Fig. 3) and dDDH values (Additional file 1: Table S4). Among the eight sequenced common bean microsymbionts, all four Ensifer strains and two Bradyrhizobium strains (Y21 and L2) displayed close relationships with soybean microsymbionts, while the other two Bradyrhizobium strains (C9 and Y36) exhibited unique genome content (Additional file 1: Table S4). Using the RecentHGT pipeline, we detected nine and 34 large-scale recent HGT events with more than 40 HGT genes among the Ensifer and Bradyrhizobium investigated, respectively. Unlike the Rhizobium strains, these common bean microsymbionts only shared HGT genes with the microsymbionts of other legume species, such as Glycine

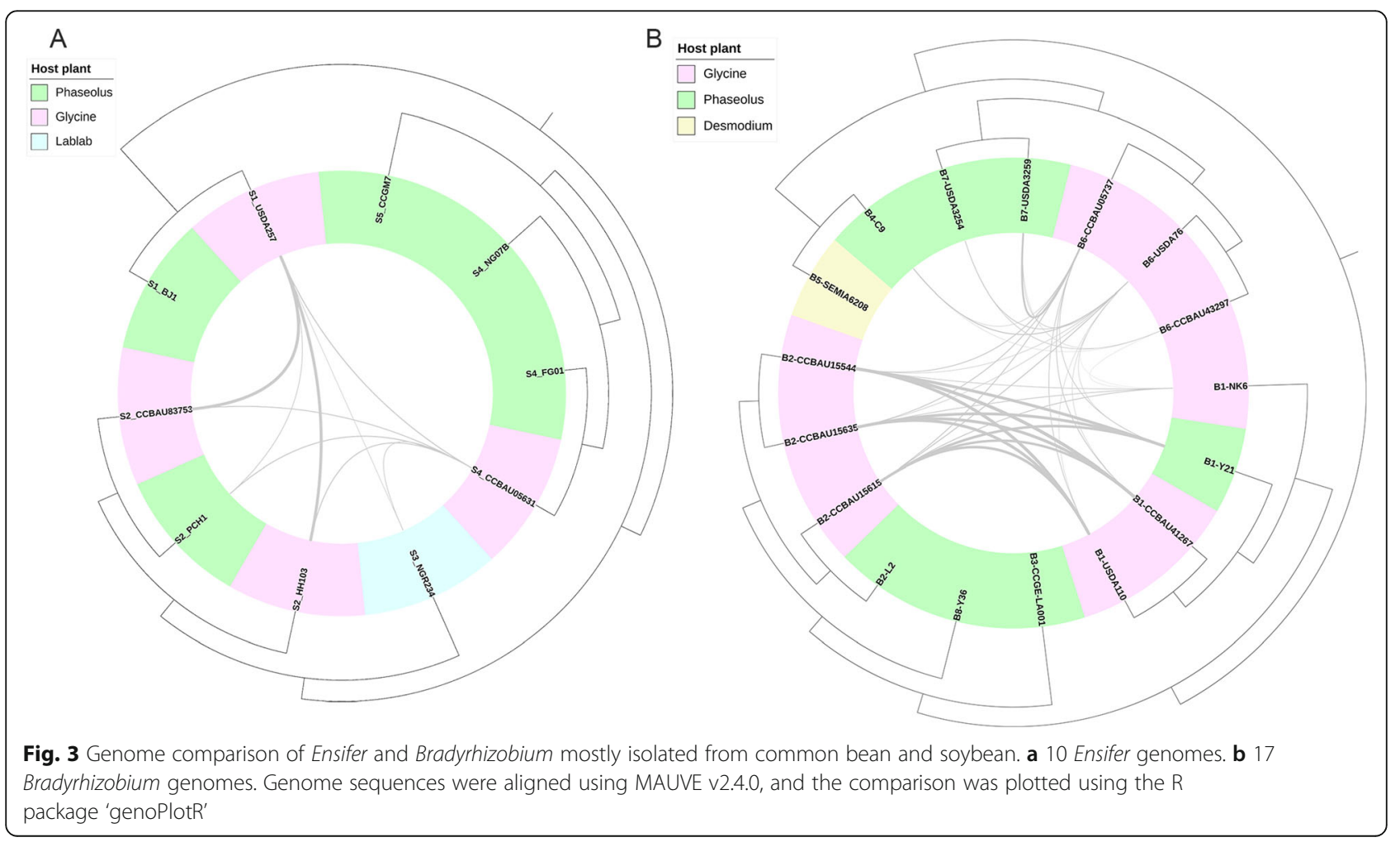


max and Lablab purpureus (Additional file 2: Figure S6; Additional file 1: Table S5).

We also analyzed 12 critical genes related to nodulation and nitrogen fixation (nodABC, fixABC, and nifHDKENB) in the eight common bean microsymbionts. Among the four Ensifer strains, Ensifer sp. III FG01 and NG07B, isolated from root nodules of common bean in Mexico, shared almost identical nodulation- and nitrogen fixationrelated genes, and these genes differed from those in Ensifer sp. III CCBAU05631 isolated from nodules of soybean. Despite their highly similar genomic background, these three strains exhibited differences in their symbiosis gene content, which could reflect host characteristics. Thus, these two symbiovars may be indicative of Ensifer sp. III. Nodulation genes of FG01/NG07B were highly similar to those of Acacia farnesiana and P. vulgaris microsymbionts, and most similar to strains with different hosts (Additional file 1: Table S6). Similarly, Ensifer sp. I BJ1 possessed heterogeneous nodulation genes from soybean microsymbionts Ensifer sp. I USDA257; however, only E. fredii PCH1 shared high similarity with nodulation genes from soybean microsymbionts $E$. fredii HH103/CCBAU83753 and USDA257/NGR234/CCBAU05631, although they were assigned to other species (Additional file 1: Table S4). Cross-nodulation tests further verified that strains PCH1, CCBAU83753, and CCBAU05631 could effectively nodulate common bean and soybean.

Among the four Bradyrhizobium strains, Bradyrhizobium sp. I L2 possessed heterogeneous nodulation genes, implying diverse sources of origin. Nodulation gene extraction failed for strain Y36, consistent with its inability to nodulate with common bean or occasional formation of rod-like and whites nodules. B. diazoefficiens Y21 and Bradyrhizobium sp. III C9 possessed different nodulation genes. Specifically, nodulation genes of strain Y21 shared high similarity with those of soybean microsymbionts $B$. diazoefficiens USDA110 $/ \mathrm{T}$ /N6/CCBAU41267 and Bradyrhizobium sp. I CCBAU15615/CCBAU15635/ CCBAU15544. Nodulation genes of strain C9 were highly similar to those of soybean microsymbionts B. elkanii CCBAU43297/CCBAU05737/USDA76. It appears that Bradyrhizobium microsymbionts of soybean possessed two sets of nodulation genes. Cross-nodulation tests revealed that both C9 and CCBAU43297 could effectively nodulate common bean and soybean while Y21, CCBAU41267, and CCBAU15615 formed white nodules with common bean and pink nodules with soybean. Strains Y21 and C9 isolated from nodules of common bean might be soybean microsymbionts. Their isolation from common bean indicates that this legume species can act as a promiscuous host.

\section{Discussion}

In this study, we sequenced rhizobial genomes from 29 common bean microsymbionts in three distinct genera,
Rhizobium, Ensifer, and Bradyrhizobium. The 29 rhizobial genomes were used to investigate the evolution of symbiotic genes among indigenous Rhizobium strains nodulating introduced bean plants, and to assess the emergence of an ability to engage in symbiotic relationships with bean plants in Bradyrhizobium and Ensifer strains.

We identified significant differences in both mean genome size and mean $\mathrm{G}+\mathrm{C}$ content among rhizobial strains in the three distinct genera (Additional file 3), in agreement with previous work [41, 45]. Functional annotation based on the COG database indicated that larger genomes might be more inclined to include genes related to three particular functional categories, namely lipid transport and metabolism (I), secondary metabolite biosynthesis, transport and catabolism $(\mathrm{Q})$, and defense mechanisms (V; Additional file 2: Figure S7). The trends of the first two categories (I and Q) are consistent with the genome analysis results of soybean microsymbionts (Ensifer and Bradyrhizobium strains) [41]. The result of the third category $(\mathrm{V})$ supports an earlier study on gene content in the genomes of 115 prokaryotic species [46]. High correlations $(|\mathrm{R}|>0.6, P<0.001$; Additional file 2: Figure S7) were obtained in approximately 30 pairs of gene functional categories; this result indicates that the functional categories complemented each other, as the identification of a series of metabolic pathways represents knowledge on the gene (molecular) interaction, reaction, and association networks [47].

Pan-genome analysis is an efficient tool for revealing the diversity and composition of the gene repertoire [48]. Herein, we used pan-genome analysis to characterize the gene repertoire of 50 available Rhizobium strains from different regions with diverse environmental conditions and host plants. The 50 Rhizobium strains were divided into 19 taxonomic clusters, with a shared core genome that represented less than half of the repertoire of proteincoding genes in each strain. Additionally, a high frequency of gene exchange with other taxa was evidenced by their large number of homologous gene in the dispensable and unique genomes. Of note, strains in the same cluster, despite isolated from diverse environments (countries or hosts), were more inclined to recruit lineage-specific shell genes under direct or indirect control through the speciation process, based on hierarchical clustering of the pangenome. Concordance between pan-genome phylogenetic tree and core genome tree was also found for Aeromonas hydrophila [49], but not for multi-genus rhizobia [41]. In addition, an open pan-genome structure has been reported for rhizobial strains of Bradyrhizobium, Ensifer [41], and Streptococcus [50]. This pan-genome structure indicates that these rhizobial genera are able to acquire exogenous DNA and/or exchange genetic material in diverse environments [51]. 
Genetic material can be acquired by bacteria via gene transfer. In vertical gene transfer, genetic information is passed from parents to offspring by asexual splitting; in the case of HGT, a donor organism transfers genes to a recipient organism that is not a descendant mainly by transformation, conjugation, and transduction [52]. Here we constructed the species tree from single-copy core genes and analyzed vertically transferred genes. Comparison with the phylogenetic tree uncovered 191 genes with phylogenies consistent with the species tree. Apart from five core genes, most of the species-related genes were detected on chromosomes. This is because plasmids are more plastic, as demonstrated in the population genomics of sympatric Rhizobium species [16]. Although most core genes have various gene histories, the coregene tree can still reflect the history of these rhizobia [53, 54]. Analysis of specific horizontally transferred genes related to the host of origin identified more genes than previously found [55]. However, a co-evolution process has been reported between nod genes and ribosomal/housekeeping genes for microsymbionts of Acacia mearnsii, indicating that microsymbionts of different host plants possess distinct evolutionary histories [56].

Based on comparison of the genomes of 50 Rhizobium strains, we assigned the 19 sequenced Chinese common bean microsymbionts to four defined species ( $R$. phaseoli, $R$. sophoriradicis, $R$. anhuiense, and $R$. esperanzae) and six putative novel species [15]. Strains in $R$. phaseoli, $R$. sophoriradicis, and $R$. esperanzae were found to be closely related to Mexican strains, not only in terms of chromosomal background, but also in symbiosis genes. Such close phylogenetic relationships indicate that the three Rhizobium species share the same origin. $R$. anhuiense may be the dominant common bean microsymbiont in China, despite the reference strain of $R$. anhuiense WYCCWR10015 has been isolated from root nodules of Trifolium repens [57], and the $R$. anhuiense type strain CCBAU23252 ${ }^{\mathrm{T}}$ is from Vicia faba [58]. In the phylogenies of 12 symbiosis genes (Additional file 2: Figure S3), we found that the topologies of strains in $R$. anhuiense (R9) were related to their host of origin. Furthermore, three symbiovars (sv. viciae, sv. phaseoli, and sv. trifolii) have been identified in this species [15]. The symbiosis plasmids of these three symbiovars in $R$. anhuiense possess different repABC operons, suggesting their independent origins. It appears that $R$. anhuiense is more flexible in terms of acquiring symbiosis plasmids. Thus, common bean microsymbionts might be microsymbionts of other indigenous legumes before the introduction of common bean into China. In addition to $R$. anhuiense, another six putative novel species were exclusive to China, although they share many recently transferred symbiosis genes with $R$. etli CFN42 ${ }^{\mathrm{T}}$. Therefore, these strains might have transitioned from microsymbionts of other legumes or soil saprophytes to bean endosymbionts. Herein, we present strong evidence that indigenous Rhizobium strains likely acquired their common bean-specific symbiosis genes from other Rhizobium species that were introduced alongside bean crops.

The transfer of symbiosis genes mediated by symbiosis plasmids from $R$. etli to other species in introduced regions appears to be a common phenomenon [40]. Herein, we did not identify any strains assigned to $R$. tropici, which is thought to be indigenous to Brazil, and which has been successfully applied for bean inoculation in Brazil [59]. Nonetheless, we identified Chinese bean microsymbionts of three defined species sharing the same origin as Mexican strains, and large-scale HGT might have occurred in seven species in the Rhizobium genus. Despite the high plasticity of symbiosis plasmids, they appear to have been transferred in an interspecies manner within the Rhizobium genus only. Our results suggest that dispersion of key symbiosis genes is rare between rhizobial generaa; however, expansion within the same genus is frequent, which could explain the emergence of multi-symbiovars [1]. In addition, we found that Ensifer and Bradyrhizobium strains possess diverse symbiotic regions compared with Rhizobium strains. Comparative genomic analysis and cross-nodulation tests of Ensifer and Bradyrhizobium strains indicated symbiotic compatibility between soybean microsymbionts and common bean microsymbionts. Nodulation genes of Ensifer strains FG01/NG07B/BJ1 and Bradyrhizobium strain L2 are heterogeneous, as they are diverse when compared with similar strains interacting with a different host.

Rhizobia can live as saprophytes in soil or establish symbiotic relationships with legumes, and they can change their host characteristics through HGT of symbiosis plasmids [60]. However, little information is available on where HGT events have occurred. This raises an interesting question: were non-symbiont strains driven to obtained "new skill" in the soil by themselves or in the nodules by plants or by other factors? Here we found that symbiosis genes in the Rhizobium genus belong to a phylogenetically compact group, although common bean can be nodulated by rhizobia from distinct genera; this result indicates that Rhizobium species exhibit a restricted host range. Overall, our findings provide insight into various aspects of rhizobial evolution, but experiments are needed to clarify the frequency of HGT of symbiosis genes among Rhizobium lineages, and the effects of host plants on transfer of these genes. HGT of specific symbiosis genes within rhizobial genera is an important mechanism that allows legumes to form symbioses with rhizobia adapted to particular soils. It is also critical to understand the strategies taken by different legume species to select particular partners. Such knowledge 
will facilitate the application of nitrogen fixation mechanisms to non-leguminous crops and thereby contribute to sustainable agriculture.

\section{Conclusions}

Among the 19 sequenced Chinese common bean microsymbionts, strains of $R$. phaseoli, $R$. sophoriradicis, and $R$. esperanzae may have been introduced together with bean seeds from Mexico. Strains of $R$. anhuiense and six putative novel Rhizobium species are exclusive to China, and they appear to have evolved into common bean microsymbionts via HGT events with Mexican rhizobia after being introduced into China. Common beannodulating Ensifer and Bradyrhizobium strains possess symbiotic regions distinct from those in Rhizobium strains, indicating symbiotic compatibility between microsymbionts of soybean and common bean. These findings provide insight into symbiotic and housekeeping genes during rhizobial evolution.

\section{Materials and methods}

Microsymbionts used in this study

We chose a total of 29 common bean microsymbionts (21 Rhizobium, four Ensifer, and four Bradyrhizobium), including 25 from China and four from Mexico. Other reference genomes used in the study included 29 Rhizobium strains (17 Phaseolus vulgaris microsymbionts, eight Trifolium spp. microsymbionts, three Mimosa spp. microsymbionts, and one Pisum sativum microsymbiont), six Ensifer strains (four soybean microsymbionts, one Phaseolus vulgaris microsymbiont, and one Lablab purpureus microsymbiont), and 13 Bradyrhizobium strains (nine soybean microsymbionts, three Phaseolus spp. microsymbionts, and one Desmodium heterocarpon microsymbiont; Additional file 1: Table S1).

\section{DNA extraction, genome sequencing, and sequence assembly}

The draft genomes of 29 common bean microsymbionts were obtained as previously described [15] using the Illumina HiSeq platform at Novogene (Beijing, China). In addition, $R$. acidisoli $\mathrm{FH} 23$ was cultured in tryptone yeast broth and genomic DNA was extracted using a DNA extraction kit (Catalogue No. 9763, TaKaRa, Dalian, China) following the manufacture's instructions. The complete genome of $R$. acidisoli FH23 was sequenced using the PacBio RS II platform at Novogene. Low-quality reads were filtered using SMRT Analysis v2.3.0 with default parameters [61], and the remaining reads were assembled to generate one contig without gaps. The genome sizes and $\mathrm{G}+\mathrm{C}$ contents of rhizobial strains in different genera were compared by Wilcoxon rank-sum tests. Genome completeness and contamination were assessed by CheckM v1.0.11 [62]. The draft genomes of 29 common bean microsymbionts and the complete genome of $R$. acidisoli FH23 have been deposited in the GenBank database under project accession no. PRJNA403813.

\section{Gene prediction and functional annotation}

A whole-genome BLAST search [63] (E-value $\leq 1 \mathrm{e}-5$, minimal alignment length percentage $\geq 40 \%$ ) was performed against the COG database [64], KEGG (Kyoto Encyclopedia of Genes and Genomes) [47], GO (Gene Ontology) [65], and NR (Non-Redundant) database. Automatic annotation was conducted on the RAST web server [66]. Correlations and $p$-values among gene functional categories and genome size were obtained using the corr.test function in the R package 'psych' and the obtained correlation matrix was visualized using the R package 'corrplot'.

\section{Orthologous gene identification and pan-genome construction}

To identify homologous gene families and construct the pan-genome of all strains, the ITEP toolkit was used for generation and curation of protein families [67]. Orthologous clusters were generated by the Markov Cluster algorithm [68], using an inflation value of 2.0 and a cutoff value of 0.4 as described previously [15]. Single-copy core genes were aligned with mafft v7.271 [69] and reversetranslated with PAL2NAL v14 [70]. Alignments were concatenated to infer maximum likelihood phylogenies with RAxML v8.2.4 under the GTR model with a gamma correction (GAMMA) for variable evolutionary rates [71]. The best maximum likelihood phylogenies were generated using autoMRE bootstrap convergence test. Presence and absence patterns in the pan-genome were statistically analyzed using an ad-hoc Python script, and the heatmap was generated using the $\mathrm{R}$ package 'pheatmap'.

\section{Plant nodulation assays, reinfection tests, and cross- nodulation tests}

Five leguminous plant species were selected for reinfection tests, namely Trifolium pratense (red clover), Mimosa pudica (mimosa), P. vulgaris (common bean), Leucaena leucocephala (white popinac), and Glycine max (soybean). More than 50 alternative strains to be selected for genome sequencing were subjected to reinfection test with common bean. In addition, cross-nodulation tests were performed using 15 representative strains, including seven sequenced Rhizobium strains (R2-L101, R2-JJW1, R4CFN42, R9-Y27, R9-WYCCWR10015, R17-FH14, and R18-FH23), three Ensifer strains (PCH1, CCBAU 83753, and CCBAU 05631), and five Bradyrhizobium strains (C9, Y21, CCBAU 43297, CCBAU 41267, and CCBAU 15615).

Briefly, leguminous plant seeds were washed with sterilized distilled water two to four times, followed by $95 \%$ ethanol $(\mathrm{v} / \mathrm{v})$ for 1-3 min. Then the seeds were surface- 
sterilized with $0.2 \% \mathrm{HgCl}_{2}$ for $3-5 \mathrm{~min}$. After rinsing and imbibition, the seeds were germinated on $0.8 \%$ water-agar plates in the dark [72]. Once the true leaves emerged, one to four seeds (based on differences in seed size and plant size) were transferred to a customized high-density polyethylene edible fungus cultivation bag (28 cm long, $7.5 \mathrm{~cm}$ wide) containing $800 \mathrm{~mL}$ of sterile vermiculite and perlite $(2: 1, \mathrm{v} / \mathrm{v})$. Each bag was inoculated with $1 \mathrm{~mL}$ of the 24 to $72 \mathrm{~h}$ culture of rhizobial strains, in triplicate. Plants were grown in a greenhouse (temperature $=28 / 20^{\circ} \mathrm{C}$, day $/$ night) and watered with $300 \mathrm{~mL}$ Fahraeus nitrogen-free mineral solution [73]. Controls were subjected to the same conditions, without rhizobial inoculation.

\section{Recent HGT detection}

The RecentHGT automatic pipeline [44] was implemented with 1137 species pairs $(\mathrm{ANI}<95 \%)$ to detect recent HGT events between two rhizobial species at the genome level and simultaneously estimate the number of transferred genes. The protein-coding sequences of all homologous genes between each species pair were retrieved from the pan-genome and aligned by the Needle tool in the EMBOSS package. The sequence similarity threshold for the expectation-maximization algorithm was set to $98.5 \%$, and the sequence similarity distribution was drawn by the 'ggplot2' package in $\mathrm{R}$.

\section{Bipartite analysis of the symbiosis plasmids}

Symbiosis plasmids were identified in 13 completely sequenced rhizobial strains nodulating $P$. vulgaris (R1CIAT652, R1-N161, R1-N671, R1-N771, R2-IE4803, R4CFN42，R6-N941，R5-N561，R5-N741，R5-N871，R6N324, R6-N541 and R18-FH23). A bipartite 'gene families-plasmids' network was constructed based on the 13 proteomes using AccNet v1.2. Specifically, all homologous proteins sharing $>40 \%$ sequence similarity were clustered with kClust parameters $-\mathrm{s}=1.73,-\mathrm{c}=0.8$, and $-\mathrm{e}=1 \mathrm{e}-10$. The bipartite graph was hierarchically clustered with a practical similarity threshold of $85 \%$. The resulting network files including nodes, edges, and clusters were then imported into Gephi v0.92 to visualize the bipartite graph [74], and the relative gene content of each plasmid was displayed by making the diameter of each node proportional to its degree. Visualization of the network was performed using Gephi's built-in ForceAtlas2 algorithm with default parameters, except for the following: approximate speed $=1.0$, scaling $=200$, gravity $=1.0$, and 'prevent overlap'.

\section{Calculation of ANI and dDDH values, and comparison of genomes}

All pairwise ANI values among the 10 Ensifer strains and 17 Bradyrhizobium strains were calculated using
JspeciesWS with the MUMmer algorithm [75], and corresponding pairwise $\mathrm{dDDH}$ values were calculated using GGDC v2.1 with the recommended Formula 2 [76]. Multiple genome alignment was performed using MAUVE v2.4.0 [77] to identify regions of sequence homology and rearrangements between the reference genome and query genome sequences. Alignments were visualized using the $\mathrm{R}$ package 'genoPlotR' [78].

\section{Supplementary information}

Supplementary information accompanies this paper at https://doi.org/10. 1186/s12864-020-6578-0.

\begin{abstract}
Additional file 1: Table S1. Genomes used in this study. Table S2. Core genes for which phylogenies are strictly consistent with the species tree. Table S3. Recent horizontal gene transfer (HGT) predictions among 50 Rhizobium strains. Table S4. Average nucleotide identity (ANI, \%) and digital DNA:DNA hybridization (dDDH, \%) values of 10 Ensifer strains and 17 Bradyrhizobium strains based on whole-genome alignments. Table S5. Recent HGT predictions among 10 Ensifer strains and 17 Bradyrhizobium strains. Table S6. Identities (\%) of nodulation- and nitrogen fixation-related genes between sequenced strains and their most closely related strains.
\end{abstract}

Additional file 2: Figure S1. Neighbor-joining species tree based on the 2110 single-copy core genes shared by 50 rhizobial genomes used in this study. The name of each strain is preceded by the cluster number in dicated in Additional file 1: Table S1. The 21 Rhizobium strains in bold font were determined in this study. Figure S2. Cross-nodulation tests on Trifolium pretense, Mimosa pudica, Phaseolus vulgaris, and Leucaena leucocephala with seven representative Rhizobium strains. * $n=3$ cultivation bags per treatment. ${ }^{* *}$ bold italic letters before strain names indicate the original host. P, Phaseolus vulgaris; T, Trifolium pretense. Figure S3. Core genes of 29 representative Rhizobium strains related to hosts. Neighborjoining trees of 12 critical symbiosis genes were constructed using 1000 bootstrap replicates (only bootstrap support $>60 \%$ is shown). Bar $=5 \%$ sequence divergence. Symbiotic characteristics are indicated by different colored solid lines (host; red = Phaseolus vulgaris, green = Mimosa spp., blue $=$ Trifolium spp.). Figure S4. Graphical circular map of the complete genome of $R$. acidisoli FH23. The outermost circle represents the coordinates of the genome sequence. Circles from outside to inside indicate protein-coding genes, genes BLAST searched against COG, KEGG, and GO databases, non-coding RNA, deviations in $\mathrm{G}+\mathrm{C}$ content (green/red), and G-C skew (light green/pink). Outermost to innermost positions indicate genes in forward and reverse orientations. Figure S5. Distribution of homologous genes based on COG assignment of unique genes within the symbiosis plasmid of R18-FH23 ( $R$. acidisoli). Figure S6. Prediction of recent HGT genes among strains isolated from different legumes. a Ensifer strains. b Bradyrhizobium strains. The width of links is proportional to the number of predicted recent HGT genes. The darker the link color, the larger the predicted magnitude of the recent HGT event. Figure S7. Correlogram showing correlations between genome size, protein-coding sequences, and COG assignments of the 29 rhizobial strains in three distinct genera. Positive correlations are indicated by cells with lower triangles or circles in upper triangles colored blue from lower left to upper right. Negative correlations are represented by red coloring from the upper left to the lower right. The darker and more saturated the color, the greater the magnitude of the correlation. Weak correlations (near zero) are almost colorless

Additional file 3. Comparison of genomic features among three distinct rhizobial genera.

\section{Abbreviations}

ANI: Average nucleotide identity; CDSs: Protein-coding sequences; dDDH: Digital DNA:DNA hybridization; HGT: Horizontal gene transfer 


\section{Acknowledgements}

We thank Dr. J. Peter W. Young for providing the genome data of Rhizobium anhuiense CCBAU 23252. We also thank Dr. Junjie Zhang for providing the culture of R. anhuiense WYCCWR10015.

\section{Authors' contributions}

WT, XL, WC, ST, and GW conceived and designed the experiments. WT performed the experiments. YC contributed the materials. WT and XL analyzed the data and drafted the manuscript. EW edited the manuscript. All authors read and approved the final manuscript.

\section{Funding}

This work was supported by the National Natural Science Foundation of China $(41830755,41671261)$ and the National Key Research \& Development Program of China (2016YFD0200308).

\section{Availability of data and materials}

The draft genomes of 29 common bean microsymbionts and the complete genome of R. acidisoli FH23 have been deposited in the GenBank database under project accession no. PRJNA403813.

\section{Ethics approval and consent to participate}

Not applicable.

\section{Consent for publication}

Not applicable.

\section{Competing interests}

The authors declare that they have no competing interests.

\section{Author details}

${ }^{1}$ State Key Laboratory of Crop Stress Biology for Arid Areas, Shaanxi Key Laboratory of Agricultural and Environmental Microbiology, College of Life Science, Northwest A\&F University, Yangling, Shaanxi 712100, People's Republic of China. ${ }^{2}$ Bioinformatics Center, Northwest A\&F University, Yangling, Shaanxi 712100, People's Republic of China. ${ }^{3}$ Departamento de Microbiología, Escuela Nacional de Ciencias Biológicas, Instituto Politécnico Nacional, 11340 México D.F, Mexico.

\section{Received: 15 May 2019 Accepted: 13 February 2020}

Published online: 27 February 2020

\section{References}

1. Remigi P, Zhu J, Young JPW, Masson-Boivin C. Symbiosis within symbiosis: evolving nitrogen-fixing legume symbionts. Trends Microbiol. 2016;24:6375 .

2. Gruber N, Galloway JN. An earth-system perspective of the global nitrogen cycle. Nature. 2008:451:293-6.

3. Schoonhoven AV, Voysest O. Common beans: research for crop improvement. Cali: CIAT; 1991.

4. Broughton WJ, Hernández G, Blair M, Beebe S, Gepts P, Vanderleyden J. Beans (Phaseolus spp.) - model food legumes. Plant Soil. 2003;252:55-128.

5. Verástegui-Valdés MM, Zhang YJ, Rivera-Orduña FN, Cheng HP, Sui XH, Wang ET. Microsymbionts of Phaseolus vulgaris in acid and alkaline soils of Mexico. Syst Appl Microbiol. 2014;37:605-12.

6. Bernal G, Graham PH. Diversity in the rhizobia associated with Phaseolus vulgaris L. in Ecuador, and comparisons with Mexican bean rhizobia. Can J Microbiol. 2001;47:526-34.

7. Grange L, Hungria M. Genetic diversity of indigenous common bean (Phaseolus vulgaris) rhizobia in two Brazilian ecosystems. Soil Biol Biochem. 2004;36:1389-98.

8. Zurdo-Piñeiro JL, García-Fraile P, Rivas R, Peix A, León-Barrios M, Willems A, et al. Rhizobia from Lanzarote, the Canary Islands, that nodulate Phaseolus vulgaris have characteristics in common with Sinorhizobium meliloti isolates from mainland Spain. Appl Environ Microbiol. 2009;75:2354-9.

9. Mnasri B, Mrabet M, Laguerre G, Aouani ME. Mhamdi RJAoM: salt-tolerant rhizobia isolated from a Tunisian oasis that are highly effective for symbiotic $\mathrm{N}_{2}$-fixation with Phaseolus vulgaris constitute a novel biovar (bv. Mediterranense) of Sinorhizobium meliloti. Arch Microbiol. 2007;187(1):79-85.

10. Mnasri B, Saïdi S, Chihaoui SA, Mhamdi R. Sinorhizobium americanum symbiovar mediterranense is a predominant symbiont that nodulates and fixes nitrogen with common bean (Phaseolus vulgaris L.) in a northern Tunisian field. Syst Appl Microbiol. 2012;35:263-9.

11. Mora Y, Díaz R, Vargaslagunas C, Peralta H, Guerrero G, Aguilar A, et al. Nitrogen-fixing rhizobial strains isolated from common bean seeds: phylogeny, physiology, and genome analysis. Appl Environ Microbiol. 2014; 80:5644-54.

12. Zhang X, Blair MW, Wang S. Genetic diversity of Chinese common bean (Phaseolus vulgaris L.) landraces assessed with simple sequence repeat markers. Theor Appl Genet. 2008;117:629-40.

13. Cao Y, Wang ET, Zhao L, Chen WM, Wei GH. Diversity and distribution of rhizobia nodulated with Phaseolus vulgaris in two ecoregions of China. Soil Biol Biochem. 2014;78:128-37.

14. Wang L, Cao Y, Wang ET, Qiao YJ, Jiao S, Liu ZS, et al. Biodiversity and biogeography of rhizobia associated with common bean (Phaseolus vulgaris L.) in Shaanxi Province. Syst Appl Microbiol. 2016;39:211-9.

15. Tong WJ, Li XC, Huo YY, Zhang L, Cao Y, Wang ET, et al. Genomic insight into the taxonomy of Rhizobium genospecies that nodulate Phaseolus vulgaris. Syst Appl Microbiol. 2018;41:300-10.

16. Pérez Carrascal OM, VanInsberghe D, Juárez S, Polz MF, Vinuesa P, González V. Population genomics of the symbiotic plasmids of sympatric nitrogenfixing Rhizobium species associated with Phaseolus vulgaris. Environ Microbiol. 2016;18:2660-76.

17. Zhang X, Turner SL, Guo X, Yang H, Debellé F, Yang G, et al. The common nodulation genes of Astragalus sinicus rhizobia are conserved despite chromosomal diversity. Appl Environ Microbiol. 2000;66:2988-95.

18. Wang S, Hao B, Li J, Gu H, Peng J, Xie F, et al. Whole-genome sequencing of Mesorhizobium huakuii 7653R provides molecular insights into host specificity and symbiosis island dynamics. BMC Genomics. 2014;15:440.

19. Galibert F, Finan TM, Long SR, Puhler A, Abola P, Ampe F, et al. The composite genome of the legume symbiont Sinorhizobium meliloti. Science. 2001;293:668-72.

20. Kaneko T, Nakamura Y, Sato S, Minamisawa K, Uchiumi T, Sasamoto S, et al. Complete genomic sequence of nitrogen-fixing symbiotic bacterium Bradyrhizobium japonicum USDA110. DNA Res. 2002;9:189-97.

21. Torres D, Revale S, Obando M, Maroniche G, Paris G, Perticari A, et al. Genome sequence of Bradyrhizobium japonicum E109, one of the most agronomically used nitrogen-fixing rhizobacteria in Argentina. Genome Announc. 2015;3:e01566-14.

22. Sullivan JT, Ronson CW. Evolution of rhizobia by acquisition of a 500-kb symbiosis island that integrates into a phe-tRNA gene. Proc Natl Acad Sci U S A. 1998;95:5145.

23. Timothy $H$, Wang $P$, Joshua $R$, Graham $O H$, Wayne $R$, John $H$, et al. Complete genome sequence of Mesorhizobium ciceri bv. Biserrulae strain WSM1284, an efficient nitrogen-fixing microsymbiont of the pasture legume Biserrula pelecinus. Genome Announc. 2016;4:e00514-6.

24. Johnston A, Beynon J, Buchanan-Wollaston A, Setchell S, Hirsch P, Beringer J. High frequency transfer of nodulating ability between strains and species of Rhizobium. Nature. 1978;276:634-6.

25. Vinuesa P, Silva C, Werner D, Martínez-Romero E. Population genetics and phylogenetic inference in bacterial molecular systematics: the roles of migration and recombination in Bradyrhizobium species cohesion and delineation. Mol Phylogenet Evol. 2005;34:29-54.

26. Ling J, Wang $\mathrm{H}$, Wu P, Li T, Tang $Y$, Naseer $\mathrm{N}$, et al. Plant nodulation inducers enhance horizontal gene transfer of Azorhizobium caulinodans symbiosis island. Proc Natl Acad Sci U S A. 2016;113:13875-80.

27. Stefanović S, Pfeil BE, Palmer JD, Doyle JJ. Relationships among phaseoloid legumes based on sequences from eight chloroplast regions. Syst Bot. 2009; 34:115-28.

28. Li Y, Guan R, Liu Z, Ma Y, Wang L, Li L, et al. Genetic structure and diversity of cultivated soybean (Glycine max (L.) Merr.) landraces in China. Theor Appl Genet. 2008;117:857-71.

29. Zhang YM, Li Y, Chen WF, Wang ET, Tian CF, Li QQ, et al. Biodiversity and biogeography of rhizobia associated with soybean plants grown in the North China plain. Appl Environ Microbiol. 2011;77:6331-42.

30. Laguerre G, Louvrier P, Allard MR, Amarger N. Compatibility of rhizobial genotypes within natural populations of Rhizobium leguminosarum biovar viciae for nodulation of host legumes. Appl Environ Microbiol. 2003;69: 2276-83.

31. Jiao Y, Liu Y, Yan H, Wang E, Tian C, Chen W, et al. Rhizobial diversity and nodulation characteristics of the extremely promiscuous legume Sophora flavescens. Mol Plant-Microbe Interact. 2015;28:1338-52. 
32. Ulrich A, Zaspel I. Phylogenetic diversity of rhizobial strains nodulating Robinia pseudoacacia L. Microbiology. 2000;146:2997-3005.

33. Moulin L, Munive A, Dreyfus B, Boivin-Masson C. Nodulation of legumes by members of the beta-subclass of Proteobacteria. Nature. 2001;411:948-50.

34. Martínez-Romero E, Segovia L, Mercante FM, Franco AA, Graham P, Pardo MA. Rhizobium tropici, a novel species nodulating Phaseolus vulgaris L. beans and Leucaena sp. trees. Int J Syst Bacteriol. 1991;41(3):417-26.

35. Wang ET, Rogel MA, García-de los Santos A, Martínez-Romero J, Cevallos MA, Martínez-Romero E. Rhizobium etli bv. mimosae, a novel biovar isolated from Mimosa affinis. Int J Syst Bacteriol. 1999;49:1479-91.

36. Geurts R, Bisseling T. Rhizobium nod factor perception and signalling. Plant Cell. 2002;14(Suppl):S239-49.

37. Jiménez-Guerrero I, Acosta-Jurado S, Del Cerro P, Pilar Navarro-Gómez P, López-Baena FJ, Ollero FJ, et al. Transcriptomic studies of the effect of nod gene-inducing molecules in rhizobia: different weapons, one purpose. Genes. 2018;9(1):1

38. Andrews M, De Meyer S, James EK, Stepkowski T, Hodge S, Simon MF, et al. Horizontal transfer of symbiosis genes within and between rhizobial genera: occurrence and importance. Genes. 2018;9:321.

39. Cao Y, Wang E, Tong W, Qiao Y, Zhao L, Chen W, et al. Population structure of Rhizobium etli-like strains nodulated with Phaseolus vulgaris in two ecoregions of China. Soil Biol Biochem. 2017;112:14-23.

40. Martínez-Romero E. Diversity of Rhizobium-Phaseolus vulgaris symbiosis: overview and perspectives. Plant Soil. 2003;252:11-23.

41. Tian CF, Zhou YJ, Zhang YM, Li QQ, Zhang YZ, Li DF, et al. Comparative genomics of rhizobia nodulating soybean suggests extensive recruitment of lineage-specific genes in adaptations. Proc Natl Acad Sci U S A. 2012;109: 8629-34.

42. Mao C, Qiu J, Wang C, Charles TC, Sobral BW. NodMutDB: a database for genes and mutants involved in symbiosis. Bioinformatics. 2005;21(12):2927-9.

43. Wang D, Yang S, Tang F, Zhu H. Symbiosis specificity in the legume: rhizobial mutualism. Cell Microbiol. 2012;14(3):334-42.

44. Li X, Tong W, Wang L, Rahman SU, Wei G, Tao S. A novel strategy for detecting recent horizontal gene transfer and its application to Rhizobium strains. Front Microbiol. 2018;9:973.

45. Ormeño-Orrillo E, Martínez-Romero E. A genomotaxonomy view of the Bradyrhizobium genus. Front Microbiol. 2019;10:1334.

46. Konstantinidis KT, Tiedje JM. Trends between gene content and genome size in prokaryotic species with larger genomes. Proc Natl Acad Sci U S A. 2004;101:3160-5.

47. Kanehisa M, Goto S, Hattori M, Aoki-Kinoshita KF, Itoh M, Kawashima S, et al. From genomics to chemical genomics: new developments in KEGG. Nucleic Acids Res. 2006;34:D354-D7.

48. Tettelin H, Riley D, Cattuto C, Medini D. Comparative genomics: the bacterial pan-genome. Curr Opin Microbiol. 2008;11:472-7.

49. Awan F, Dong Y, Liu J, Wang N, Mushtaq MH, Lu C, et al. Comparative genome analysis provides deep insights into Aeromonas hydrophila taxonomy and virulence-related factors. BMC Genomics. 2018;19:712.

50. Donati C, Hiller NL, Tettelin H, Muzzi A, Croucher NJ. Angiuoli SV, et al structure and dynamics of the pan-genome of Streptococcus pneumoniae and closely related species. Genome Biol. 2010;11:R107.

51. Rouli L, Merhej V, Fournier PE, Raoult D. The bacterial pangenome as a new tool for analysing pathogenic bacteria. New Microbes New Infect. 2015;7: 72-85.

52. Gogarten JP, Townsend JP. Horizontal gene transfer, genome innovation and evolution. Nat Rev Microbiol. 2005;3:679.

53. Xie JB, Du Z, Bai L, Tian C, Zhang Y, Xie J, et al. Comparative genomic analysis of $\mathrm{N}_{2}$-fixing and non- $\mathrm{N}_{2}$-fixing Paenibacillus spp.: organization, evolution and expression of the nitrogen fixation genes. PLoS Genet. 2014; 10:e1004231.

54. Haggerty LS, Martin FJ, Fitzpatrick DA, Mclnerney JO. Gene and genome trees conflict at many levels. Philos Trans R Soc Lond Ser B Biol Sci. 2009; 364:2209-19.

55. Clúa J, Roda C, Zanetti M, Blanco F. Compatibility between legumes and rhizobia for the establishment of a successful nitrogen-fixing symbiosis. Genes. 2018;9:125.

56. Menna P, Hungria M. Phylogeny of nodulation and nitrogen-fixation genes in Bradyrhizobium: supporting evidence for the theory of monophyletic origin, and spread and maintenance by both horizontal and vertical transfer. Int J Syst Evol Microbiol. 2011;61:3052-67.
57. Zhang JJ, Jing XY, Lajudie PD, Ma C, He PX, Singh RP, et al. Association of white clover (Trifolium repens L.) with rhizobia of sv. Trifolii belonging to three genomic species in alkaline soils in north and East China. Plant Soil. 2016:407:417-27.

58. Zhang YJ, Zheng WT, Everall I, Young JP, Zhang XX, Tian CF, et al. Rhizobium anhuiense sp. nov., isolated from effective nodules of Vicia faba and Pisum sativum. Int J Syst Evol Micr. 2015;65:2960-7.

59. Grange L, Hungria M, Graham PH, Martínez-Romero E. New insights into the origins and evolution of rhizobia that nodulate common bean (Phaseolus vulgaris) in Brazil. Soil Biol Biochem. 2007;39:867-76.

60. Poole P, Ramachandran V, Terpolilli J. Rhizobia: from saprophytes to endosymbionts. Nat Rev Microbiol. 2018;16:291-303.

61. Berlin K, Koren S, Chin CS, Drake JP, Landolin JM, Phillippy AM. Assembling large genomes with single-molecule sequencing and locality-sensitive hashing. Nat Biotechnol. 2015;33:623-30.

62. Parks DH, Imelfort M, Skennerton CT, Hugenholtz P, Tyson GW. CheckM: assessing the quality of microbial genomes recovered from isolates, single cells, and metagenomes. Genome Res. 2015;25:1043-55.

63. Altschul SF, Gish W, Miller W, Myers EW, Lipman DJ. Basic local alignment search tool. J Mol Biol. 1990;215:403-10.

64. Tatusov RL, Fedorova ND, Jackson JD, Jacobs AR, Kiryutin B, Koonin EV, et al. The COG database: an updated version includes eukaryotes. BMC Bioinformatics. 2003;4:41.

65. Ashburner M, Ball CA, Blake JA, Botstein D, Butler H, Cherry JM, et al. Gene ontology: tool for the unification of biology. Nat Genet. 2000;25:25-9.

66. Aziz RK, Bartels D, Best AA, Dejongh M, Disz T, Edwards RA, et al. The RAST server: rapid annotations using subsystems technology. BMC Genomics. 2008;9:75.

67. Benedict MN, Henriksen JR, Metcalf WW, Whitaker RJ, Price ND. ITEP: an integrated toolkit for exploration of microbial pan-genomes. BMC Genomics. 2014;15:112-20.

68. Enright AJ, Van Dongen S, Ouzounis CA. An efficient algorithm for largescale detection of protein families. Nucleic Acids Res. 2002;30:1575-84.

69. Katoh K, Standley DM. MAFFT multiple sequence alignment software version 7: improvements in performance and usability. Mol Biol Evol. 2013; 30:772-80.

70. Suyama M, Torrents D, Bork P. PAL2NAL: robust conversion of protein sequence alignments into the corresponding codon alignments. Nucleic Acids Res. 2006;34:W609-12.

71. Stamatakis A. RAxML version 8: a tool for phylogenetic analysis and postanalysis of large phylogenies. Bioinformatics. 2014;30:1312-3.

72. Caetanoanollés G, Favelukes G, Bauer WD. Optimization of surface sterilization for legume seed. Comp Stand Inter. 1990;31:261-71.

73. Fahraeus $\mathrm{G}$. The infection of clover root hairs by nodule bacteria studied by a simple glass slide technique. J Gen Microbiol. 1957;16:374-81.

74. Bastian M, Heymann S, Jacomy M. Gephi: an open source software for exploring and manipulating networks. ICWSM: The AAAI Press; 2009.

75. Richter M, Rossello-Mora R, Oliver Glockner F, Peplies J. JSpeciesWS: a web server for prokaryotic species circumscription based on pairwise genome comparison. Bioinformatics. 2016;32:929-31.

76. Meierkolthoff JP, Auch AF, Klenk HP, Göker M. Genome sequence-based species delimitation with confidence intervals and improved distance functions. BMC Bioinformatics. 2013;14:60.

77. Darling AE, Mau B, Perna NT. ProgressiveMauve: multiple genome alignment with gene gain, loss and rearrangement. PLoS One. 2010;5: e11147.

78. Guy L, Roat Kultima J, Andersson SGE. GenoPlotR: comparative gene and genome visualization in R. Bioinformatics. 2010;26:2334-5.

\section{Publisher's Note}

Springer Nature remains neutral with regard to jurisdictional claims in published maps and institutional affiliations. 